\title{
Automated freeze-thaw cycles for decellularization of tendon tissue - a pilot study
}

Susanne Pauline Roth ${ }^{1,2^{*}}$, Sina Marie Glauche ${ }^{2}$, Amelie Plenge ${ }^{3}$, Ina Erbe ${ }^{1}$, Sandra Heller ${ }^{4}$ and Janina Burk ${ }^{1,2,5}$

\begin{abstract}
Background: Decellularization of tendon tissue plays a pivotal role in current tissue engineering approaches for in vitro research as well as for translation of graft-based tendon restoration into clinics. Automation of essential decellularization steps like freeze-thawing is crucial for the development of more standardized decellularization protocols and commercial graft production under good manufacturing practice (GMP) conditions in the future.

Methods: In this study, a liquid nitrogen-based controlled rate freezer was utilized for automation of repeated freeze-thawing for decellularization of equine superficial digital flexor tendons. Additional tendon specimens underwent manually performed freeze-thaw cycles based on an established procedure. Tendon decellularization was completed by using non-ionic detergent treatment (Triton X-100). Effectiveness of decellularization was assessed by residual nuclei count and calculation of DNA content. Cytocompatibility was evaluated by culturing allogeneic adipose tissue-derived mesenchymal stromal cells on the tendon scaffolds.
\end{abstract}

Results: There were no significant differences in decellularization effectiveness between samples decellularized by the automated freeze-thaw procedure and samples that underwent manual freeze-thaw cycles. Further, we inferred no significant differences in the effectiveness of decellularization between two different cooling and heating rates applied in the automated freeze-thaw process. Both the automated protocols and the manually performed protocol resulted in roughly $2 \%$ residual nuclei and $13 \%$ residual DNA content. Successful cell culture was achieved with samples decellularized by automated freeze-thawing as well as with tendon samples decellularized by manually performed freeze-thaw cycles.

Conclusions: Automated freeze-thaw cycles performed by using a liquid nitrogen-based controlled rate freezer were as effective as previously described manual freeze-thaw procedures for decellularization of equine superficial digital flexor tendons. The automation of this key procedure in decellularization of large tendon samples is an important step towards the processing of large sample quantities under standardized conditions. Furthermore, with a view to the production of commercially available tendon graft-based materials for application in human and veterinary medicine, the automation of key procedural steps is highly required to develop manufacturing processes under GMP conditions.

Keywords: Regenerative medicine, Tissue engineering, Tendon, Horse, Automation, Decellularization, Controlled rate freezer

\footnotetext{
* Correspondence: susanne.roth@uni-leipzig.de

'Large Animal Clinic for Surgery, University of Leipzig, An den Tierkliniken 21, Leipzig 04103, Germany

${ }^{2}$ Saxonian Incubator for Clinical Translation, University of Leipzig,

Philipp-Rosenthal-Strasse 55, Leipzig 04103, Germany

Full list of author information is available at the end of the article
} 


\section{Background}

Decellularization of natural tissues offers promising opportunities as a multi-purpose tool in the field of tissue engineering [1]. Particularly, the challenging creation of decellularized biological scaffolds with a preserved tissuespecific extracellular matrix (ECM) composition provides a crucial field of application. The importance of biological scaffolds with a naturally structured ECM is based on their similarity to in vivo conditions for cell attachment, proliferation, and differentiation while maintaining biomechanical functionality and biocompatibility.

Among biological scaffolds used for clinical application in regenerative medicine and for current tissue engineering approaches, decellularized tendon tissue plays a pivotal role. This is due to a high and still increasing incidence of tendon pathologies, such as injuries of the Achilles tendon and traumatic rupture of the anterior cruciate ligament, in an aging population with growing sporting ambitions [2-4]. Moreover, currently available treatment options for tendinopathies are often not evidence-based and do not effectively prevent re-injury, placing a heavy burden on the health care system and the social economy [5]. Therefore, tissue engineering involving decellularization techniques aims not only to prepare 3D-culture models for in vitro research [6], but also to produce clinically available tissue grafts for the reconstruction of musculoskeletal defects $[1,7]$ with the ultimate goal to translate tendon restoration into clinics.

The distinguishing and so far inimitable feature of site-specific homologous tissue for graft-based tendon reconstruction is its highly specific ECM composition, reflecting original biochemical and biomechanical tissue characteristics at its best. Since synthetic biomaterials turned out to be more or less inadequate for tendon reconstruction, biological tendon grafts including auto-, allo- and xenografts are considered as gold standard for tendon repair $[1,5]$. Especially due to donor site morbidity, abundantly available allo- and xenografts are favoured for the development of scaffolds reflecting natural tendon ECM composition [1, 8]. In order to avoid rejection after implantation due to cell associated immune response, and to allow re-seeding procedures, decellularization is an essential step towards the application of appropriate tendon-derived scaffolds [8].

To date, different protocols for decellularization of tendon tissue of various species have been evaluated, using physical as well as chemical and/or enzyme-based methods. However, published protocols for decellularization of natural tendon tissue provide little insight regarding the impact of specific decellularization steps [9]. Physical treatments, like agitation or sonication, mechanical massage or pressure, or freeze-thaw cycles, are the most commonly used techniques to disrupt cell membranes, detach cells within their ECM network and allow further rinsing to remove cell remnants [5, 10]. Whereas the sole application of freeze-thawing was reported as insufficient for tendon decellularization [10], Burk et al. reported a significantly more effective decellularization of large tendon samples by combining repetitive freeze-thaw cycles with a detergent treatment when compared to a sole application of detergents [11]. Although procedural steps such as freeze-thaw cycles are considered as key procedure, there is no consensus on a certain protocol or standardized parameters for evaluation of their effectiveness [12-14].

Considering safety of application in clinical use and the necessity of commercial graft production under good manufacturing practice (GMP) conditions, standardization and accurate documentation of all procedural steps are crucial. Furthermore, identical processing of large sample quantities will be required, which can only be achieved by automating key procedures of decellularization. Therefore, our study aimed to directly compare the influence of manual and automated freeze-thaw cycles on the decellularization effectiveness in equine superficial digital flexor tendons, based on a previously described decellularization protocol [11]. Further, we intended to evaluate the impact of two different cooling as well as heating rates in automated freeze-thaw cycles with regard to decellularization effectiveness. To our knowledge, this is the first description of using automated freeze-thaw cycles for tissue decellularization, leading to standardization and optimization of decellularization protocols.

\section{Methods}

\section{Study design}

Freshly collected equine superficial digital flexor tendon samples $(n=10)$ were used to assess the effectiveness of automated freeze-thawing as part of an otherwise established decellularization protocol. Two different freezethawing protocols were carried out using a controlled rate freezer (PLANER ${ }^{\oplus}$ Kryo 360 - 1.7). Additionally, a manual freeze-thawing procedure in accordance with an established protocol was done.

In all three protocols (Auto-Protocol 1 and 2 and Manual-Protocol, respectively), after decellularization was initiated by repetitive freeze-thaw cycles, the same subsequent procedure of detergent treatment was applied. Parameters to evaluate the effectiveness of decellularization included histologically visible nuclei and DNA content. In addition, further tendon samples $(n=3)$ were re-seeded with allogeneic equine adipose tissue-derived mesenchymal stromal cells (AT-MSC) to evaluate cytocompatibility.

\section{Sample collection}

Fresh cadaver limbs from middle-sized warmblood horses obtained at a local abattoir served as source of tendon samples. The aseptically performed collection of 
tendon specimens from the palmar/plantar aspect of the mid-metacarpus/-metatarsus was done within $6 \mathrm{~h}$ after slaughter under sterile working conditions. During this period of time, the skin of the cadaver limbs remained closed to keep the tendon tissue sterile. To further prevent bacterial contamination, the recovered tendon samples were placed in phosphate buffered saline (PBS; Sigmal Aldrich) supplemented with $2 \%$ penicillinstreptomycin (Sigma Aldrich) and $0.1 \%$ gentamycin (Carl Roth) for overnight storage at $4{ }^{\circ} \mathrm{C}$. Immediately before automated or manual freeze-thawing, the stored tendon specimens were washed 5 min each in PBS and 70\% ethanol two times.

\section{Decellularization}

From each $8 \mathrm{~cm}$ long tendon sample, $2 \mathrm{~cm}$ were separated to serve as internal control, whereas the remaining $6 \mathrm{~cm}$ were divided into three equal $2 \mathrm{~cm}$ long parts. The cross-sectional dimension of the tendons were left unchanged, measuring roughly $1.5 \mathrm{~cm} \times 0.5 \mathrm{~cm}$. Each of the latter tendon parts underwent decellularization according to one of three different protocols (Table 1). In group 1 and 2 (Auto-Protocol 1 and 2), automated freeze-thaw cycles with controlled cooling and heating rates differing in the applied temperature change per minute were performed. Safe and sterile placement of tendon samples inside the controlled rate freezer was ensured by using plastic sampling bags (Carl Roth; $n=7$ tendon samples) or $15 \mathrm{ml}$ conical centrifuge tubes (VWR; $n=3$ tendon samples). For this purpose, the tube holders of the controlled rate freezer had been modified in collaboration with the manufacturer. Group 3 samples (Manual-Protocol) were subjected to manual freezethaw cycles including five cycles of 2 min freezing in liquid nitrogen and $10 \mathrm{~min}$ thawing in PBS at $37{ }^{\circ} \mathrm{C}$. Further decellularization was carried out at room temperature as well as under continuous agitation and was performed in the same way for all three groups. For the purpose of rinsing and induction of cell lysis by osmotic effects, all samples were incubated in hypotonic solution (distilled water) for $48 \mathrm{~h}$. Afterwards, tendon samples were incubated for $48 \mathrm{~h}$ in Tris buffer (Carl Roth) (pH 7.6) containing 1\% Triton X-100 (Carl Roth). Decellularization was completed by the following washing steps: $2 \times 15 \mathrm{~min}$ in distilled water, $24 \mathrm{~h}$ in cell culture medium [DMEM $1 \mathrm{~g}$ glucose/L (Thermo Fisher Scientific) supplemented with $10 \%$ fetal bovine serum (FBS; Sigma Aldrich), 1\% penicillin-streptomycin (Sigma Aldrich), 0.1\% gentamycin (Carl Roth)], and again $24 \mathrm{~h}$ in PBS. The performed incubation in cell culture medium served not only the purpose of rinsing to remove cellular remnants and residual chemicals, but also to prepare the scaffolds for cell culture optimally.

\section{Assessment of decellularization effectiveness Histology and nuclei count}

For this analysis, one piece of tendon tissue was obtained from the centre of each sample. These pieces were fixed in $4 \%$ paraformaldehyde and embedded in paraffin. Hematoxylin and eosin staining of two longitudinal $6 \mu \mathrm{m}$ sections per sample followed. Three randomly chosen regions of the prepared slides were photographed at $20 \times$ magnification (Leica DMi1, Leica MC 170HD, LAS V4.5 Software, Leica Microscope CMS GmbH) and visible cell nuclei were counted. Results from visible nuclei count were normalized to the respective internal controls and are given as percentages relative to controls.

\section{DNA content}

To calculate DNA content, pieces of tendon tissue recovered from the centre of each sample were subjected to papain digestion. For this purpose, $200 \mathrm{mg}$ of each sample were minced into $1 \mathrm{~mm}^{3}$ pieces and washed in PBS. Subsequently, the samples were incubated in $800 \mu \mathrm{l}$ papain digestion buffer $[2 \mathrm{mM} \mathrm{n}$-acetyl-l-cysteine (Sigma Aldrich), $2 \mathrm{mM}$ EDTA (Carl Roth), $50 \mathrm{mM} \mathrm{Na} \mathrm{HPO}_{4}$ (Carl Roth)] and $20 \mu \mathrm{l}$ papain solution $(10 \mathrm{mg} / \mathrm{ml})$

Table 1 Sample groups and decellularization protocols

\begin{tabular}{|c|c|c|c|c|c|c|}
\hline \multirow[t]{3}{*}{ Group } & \multirow[t]{3}{*}{ Protocol } & \multicolumn{5}{|c|}{ Decellularization procedures } \\
\hline & & \multicolumn{4}{|c|}{5 repetitions of freeze-thawing } & \multirow[t]{2}{*}{ Further treatment } \\
\hline & & Cooling & Freeze hold & Heating & Thaw hold & \\
\hline 1 & Auto-Protocol 1 & $-50{ }^{\circ} \mathrm{C}$ per min & $3 \mathrm{~min}$ at $-80^{\circ} \mathrm{C}$ & $+50^{\circ} \mathrm{C}$ per min & 10 min at $+20^{\circ} \mathrm{C}$ & \multirow{4}{*}{$\begin{array}{l}48 \text { h distilled water } \\
48 \text { h } 1 \% \text { Triton X-100 } \\
\text { Washing steps }\end{array}$} \\
\hline 2 & Auto-Protocol 2 & $-20{ }^{\circ} \mathrm{C}$ per min & 3 min at $-80^{\circ} \mathrm{C}$ & $+20^{\circ} \mathrm{C}$ per min & $10 \mathrm{~min}$ at $+20^{\circ} \mathrm{C}$ & \\
\hline \multirow[t]{2}{*}{3} & Manual-Protocol & Manual transfer & 2 min in liquid nitrogen & Manual transfer & $10 \mathrm{~min}$ in $37^{\circ} \mathrm{CPBS}$ & \\
\hline & Control & No treatment & & & & \\
\hline
\end{tabular}

Equine superficial digital flexor tendon samples of group 1 and group 2 were processed by automated freeze-thaw cycles, differing in the performed cooling and heating rates (Auto-Protocol 1 and Auto-Protocol 2). Both of the applied cooling and heating rates describe a temperature change per unit time. For Auto-Protocol 1 as well as for Auto-Protocol 2 the maximum reached temperature was $+20^{\circ} \mathrm{C}$ (thaw hold for $10 \mathrm{~min}$ ) and the minimum reached temperature was $-80{ }^{\circ} \mathrm{C}$ (freeze hold for 3 min). All

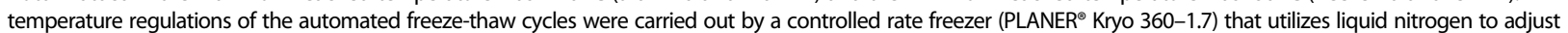
temperature. Group 3 included manual freeze-thaw cycles. Further steps of decellularization were the same for all sample groups. Tendon samples classified as internal control underwent no decellularization 
(Sigma Aldrich) for $24 \mathrm{~h}$ at $60{ }^{\circ} \mathrm{C}$. Storage of digested tissue samples before further analysis took place at $-20^{\circ} \mathrm{C}$.

DNA content was measured using the Quant-IT ${ }^{\mathrm{mm}}$ PicoGreen ${ }^{\circ}$ dsDNA assay kit (Thermo Fisher Scientific). To achieve this, digested tissue samples (10-fold dilution for internal controls) were pipetted in 96-well plates and an equal volume of PicoGreen ${ }^{\circ}$ reagent working solution was added. Subsequently, the samples were incubated for $5 \mathrm{~min}$ at room temperature, protected from light. Fluorescence (excitation wavelength of $480 \mathrm{~nm}$ ) was measured by a microplate reader (SynergyTM H1 Hybrid Multi-Mode Microplate Reader, Gen5TM Software, BioTek $^{\ominus}$ Instruments, Inc.). DNA content was determined by the use of standard curves prepared from DNA standards measured on the same plates.

Results from DNA measurement were again normalized to the respective controls and are given as percentages relative to controls.

\section{Assessment of cytocompatibility Scaffold seeding}

Allogeneic equine AT-MSC were isolated by enzymatic digestion with collagenase I (Thermo Fisher Scientific) and expanded until passage 3. Plastic-adherence, trilineage differentiation potential and expression of MSC-related surface markers were shown before further processing of the cells (data not shown). Cells cultured to approximately $60-80 \%$ of confluence were detached by trypsinization and suspended in cell culture medium for re-seeding experiments.

Scaffolds with a $2 \mathrm{~cm}^{2}$ surface $(2 \mathrm{~cm} \times 1 \mathrm{~cm} \times 2 \mathrm{~mm})$ from tendons that were decellularized by automated and manual protocols as described above were manually prepared using stirrup-shaped blades (Carl Roth). AT-MSC were then seeded onto the surface of the tendon scaffolds (130,000 cells in $30 \mu \mathrm{l} / \mathrm{cm}^{2}$ scaffold surface). After incubation at $37{ }^{\circ} \mathrm{C}$ and $5 \% \mathrm{CO}_{2}$ for $4 \mathrm{~h}$, all seeded tendon scaffolds were covered with cell culture medium and further incubated for $3 \mathrm{~d}$ at $37^{\circ} \mathrm{C}$ and $5 \% \mathrm{CO}_{2}$.

\section{Histology and LIVE/DEAD ${ }^{\circledR}$ staining}

To assess cell morphology and cell integration into the scaffold, hematoxylin and eosin staining of paraffin sections of the seeded scaffolds was performed as described above. Further, LIVE/DEAD ${ }^{\circ}$ staining of seeded scaffolds was carried out using the staining kit [LIVE/DEAD ${ }^{\circ}$ Viability/Cytotoxicity Kit, for mammalian cells, Thermo Fisher Scientific; calcein AM $4 \mathrm{mM}$ in anhydrous DMSO, ethidium homodimer-I $2 \mathrm{mM}$ in DMSO/ $\mathrm{H}_{2} \mathrm{O} 1$ : $4(\mathrm{v} / \mathrm{v})]$ according to the manufacturer's instructions. The latter was used to evaluate cell viability and morphology as well as alignment and distribution of the seeded cells on the scaffold surface.

\section{Statistical analysis}

Statistical data analysis was performed using SPSS $^{\bullet}$ Statistics 22.0. For comparison of parameters among different groups, Friedman tests were used. Further analysis by Wilcoxon signed-rank tests was then carried out for paired comparisons. As there were no significant differences between tendon samples that underwent automated freeze-thaw cycles encased either by the plastic sampling bags or by the $15 \mathrm{ml}$ centrifuge tubes, evaluation of decellularization effectiveness was performed regardless of the sample holder used. The level of significance was defined at $p=0.05$.

\section{Results}

Performance of the controlled rate freezer

Graphical records printed by the controller of the controlled rate freezer plotted the actual measured temperature profile of Auto-Protocol 1 or 2 versus the programmed temperature profile (Fig. 1). The controlled rate freezer repeatably reached the required set point temperatures within the programmed period of time and with good accuracy in Auto-Protocol 2. However, in Auto-Protocol 1, which included faster cooling and heating rates, a growing delay was repeatedly observed compared to the programmed temperature profile. Furthermore, short-term temperature over- or undershoots were constantly evident at the beginning of each programmed hold at the required set point temperatures. This effect was moderate for Auto-Protocol 2 but more obvious in the records for Auto-Protocol 1.

\section{Effectiveness of decellularization}

All of the performed protocols resulted in both reduced cell nuclei count and reduced DNA content $(p=0.005)$ relative to respective controls, with no significant differences among samples from the three different freezethaw procedures (Fig. 2).

Histologically, we observed very few visible nuclei within a uniformly structured ECM (Fig. 3). Empty gaps were observed, free of cellular residues, between regularly aligned collagen fibres. In contrast, histologically evaluated control scaffolds showed a preserved cellular integrity with rarely occurring signs of apoptosis. Normalized percentages of residual nuclei in tendon samples treated with Auto-Protocol 1 (mean value: 2.6\%; range: $0-11.9 \%$ ) and 2 (mean value: $2.5 \%$; range: $0-6.1 \%$ ) were slightly higher than those treated manually (mean value: $1.6 \%$; range: $0-4.3 \%$ ).

Mean values of residual DNA (normalized percentage values) for all applied protocols fall within a narrow range of 12.8\% (Auto-Protocol 1; range: 9.2-18.2\%) and 13.7\% (Manual Protocol; range: 8.3-18.9\%; AutoProtocol 2; range: 9.3-16.3\%). The obtained data for 


\section{a}

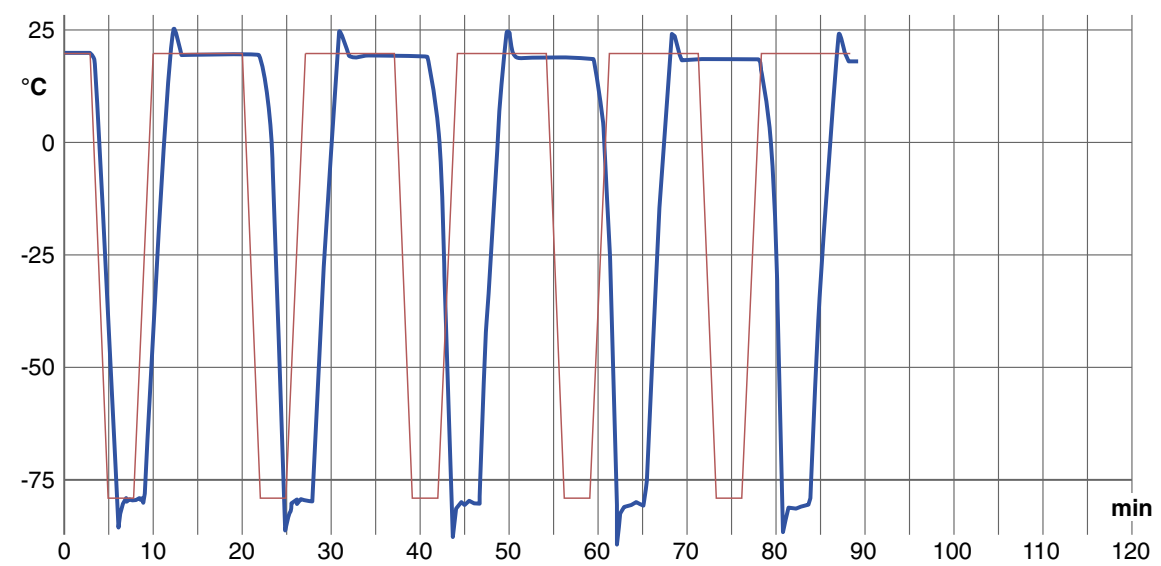

b

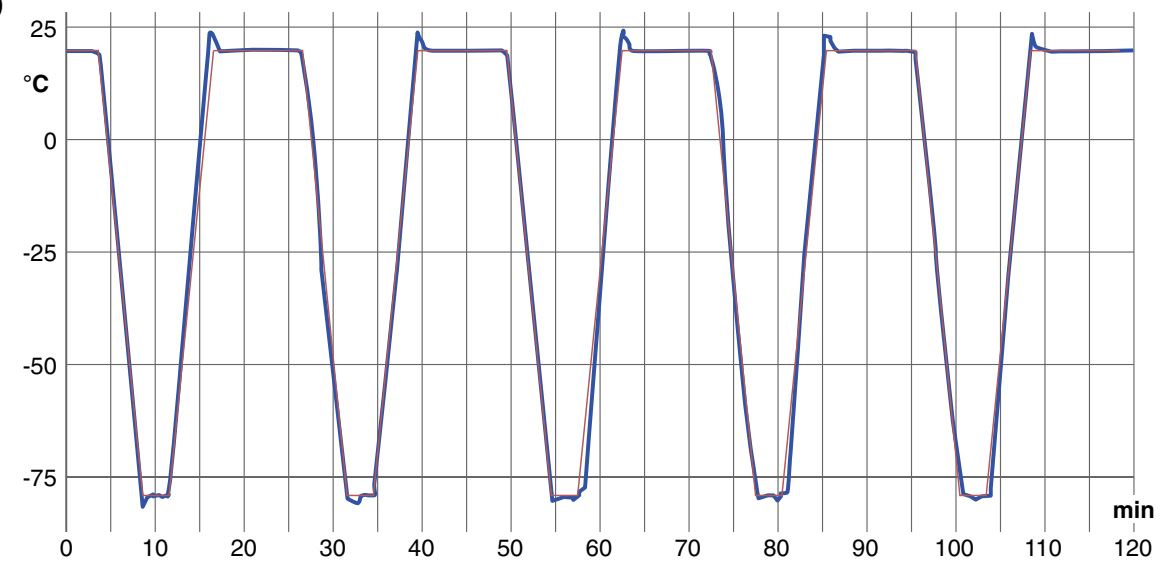

Fig. 1 Temperature profiles of Auto-Protocol 1 (group 1) (a) and Auto-Protocol 2 (group 2) (b). Representative graphics for group 1 (a) (Auto-Protocol 1; cooling/heating rate of $50^{\circ} \mathrm{C} / \mathrm{min}$ ) and for group 2 (b) (Auto-Protocol 2; cooling/heating rate of $20^{\circ} \mathrm{C} / \mathrm{min}$ ). Blue curves represent actual values and brown curves show target values of the temperature. Both graphics are prepared on the basis of printed temperature records of the biological controlled rate freezer (PLANER ${ }^{\circledast}$ Kryo 360-1.7) by the use of Adobe ${ }^{\circledast}$ Illustrator ${ }^{\circledast}$ CS6 software
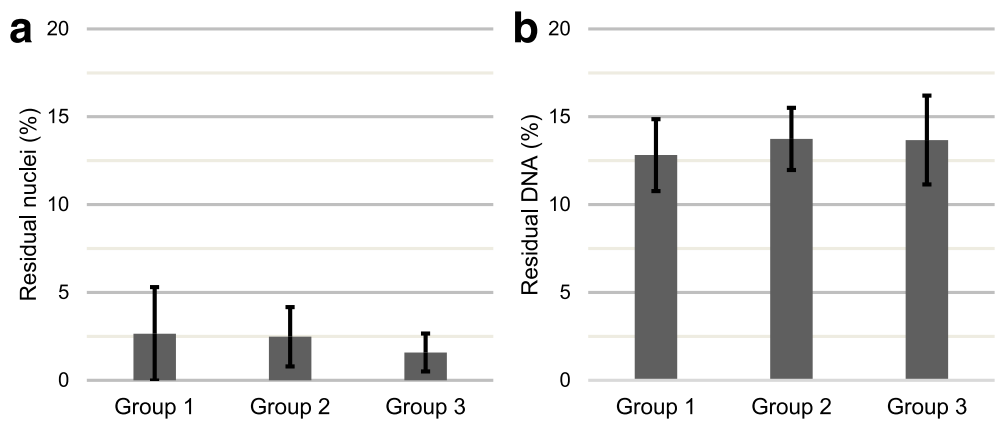

Fig. 2 Visible nuclei count (a) and DNA content (b) of decellularized tendon samples ( $n=10)$. Mean values of residual nuclei count (a) and residual DNA (b) in \% relative to the controls $(n=10)$. The vertical error bars indicate the confidence interval of $95 \%$. There were no significant differences in the number of residual nuclei and in the amount of DNA content among tendon samples of both automated protocols (group 1 and 2) and the manually performed protocol (group 3) for decellularization 

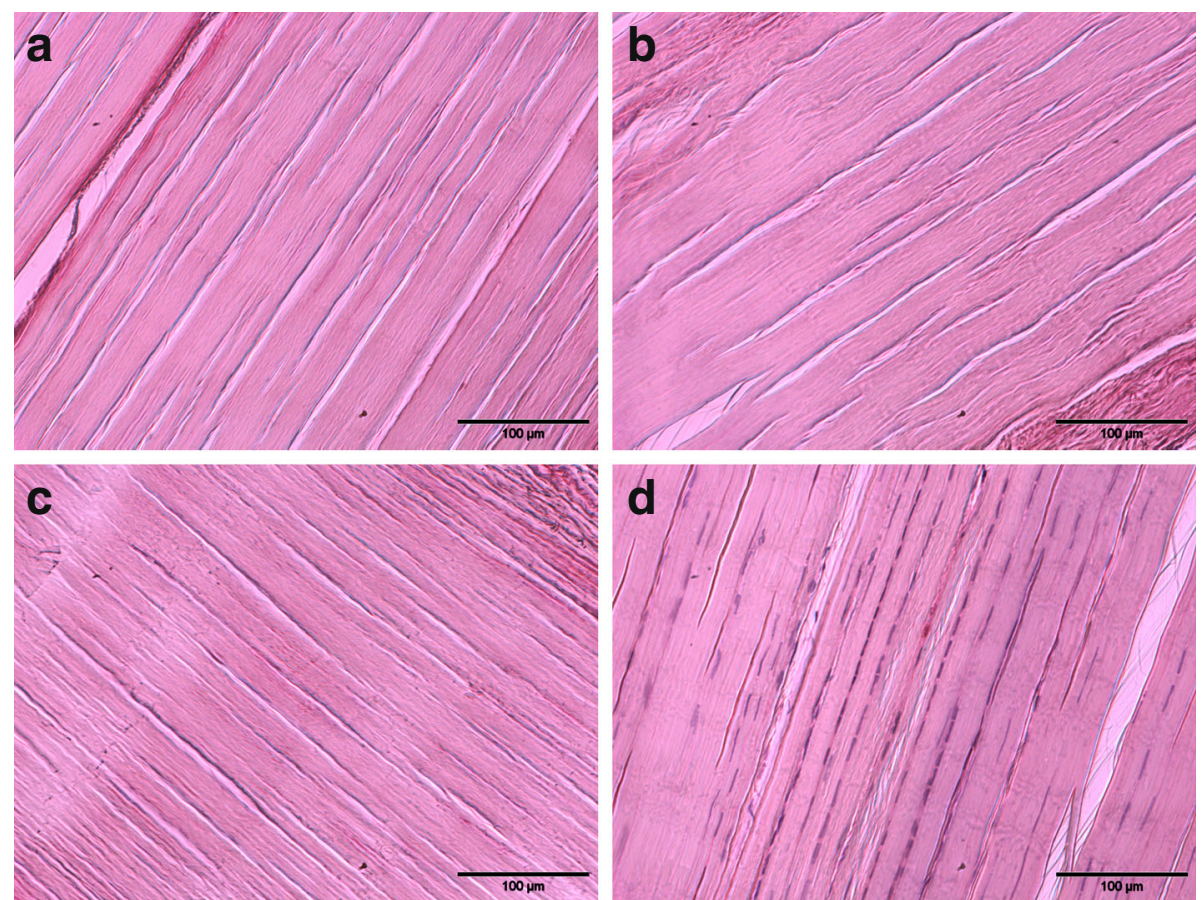

Fig. 3 Histological assessment of decellularization effectiveness. Representative images of hematoxylin and eosin stained equine superficial digital flexor tendon samples of group 1 (Auto-Protocol 1) (a), group 2 (Auto-Protocol 2) (b), group 3 (Manual Protocol) (c), showing an apparent reduction of visible nuclei compared with tendon samples of the internal controls (no decellularization) (d). Decellularized tendon samples of all groups reveal regularly aligned collagen fibrils and interfibrillar tissue gaps instead of resident cells

decellularized tendon samples correspond to a range from 9.7 to $12.7 \mathrm{ng} \mathrm{DNA} / \mathrm{mg}$ wet weight.

\section{Cytocompatibility}

Hematoxylin and eosin staining of re-seeded tendon scaffolds revealed successful seeding on the surface of automatically and manually processed scaffolds (Fig. 4). At day 3 after re-seeding, the majority of visible cells were attached to the scaffold surface, only a very low number of cells populated deeper tissue layers.
LIVE/DEAD staining of re-seeded scaffolds showed that the majority of the cells was vital, indicated by green fluorescence, and a lower number of cells with damaged membranes indicated by red fluorescence. Morphologically, vital cells appeared elongated with collagen fibre oriented alignment, whereas roundly shaped, damaged cells showed no tendency for directed orientation (Fig. 5). The observed cell distribution was slightly inhomogeneous. Besides areas with an even distribution, there were also only sparsely populated parts of the scaffold surface.
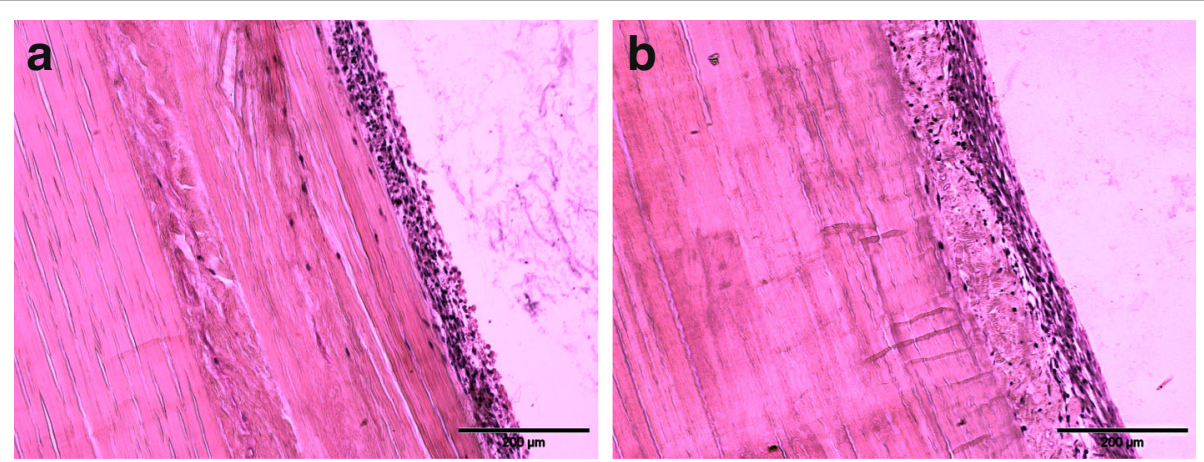

Fig. 4 Histological assessment of cytocompatibility. Representative images of hematoxylin and eosin stained equine superficial digital flexor tendon samples after decellularization by automated (a) and manual (b) freeze-thaw cycles, re-seeding with equine adipose tissue-derived mesenchymal stromal cells and 3 days of culture. A successful re-seeding procedure of the tendon surface is indicated by the dense cell layer adhering to the sample surface, with a lower number of cells penetrating deeper tissue structures 


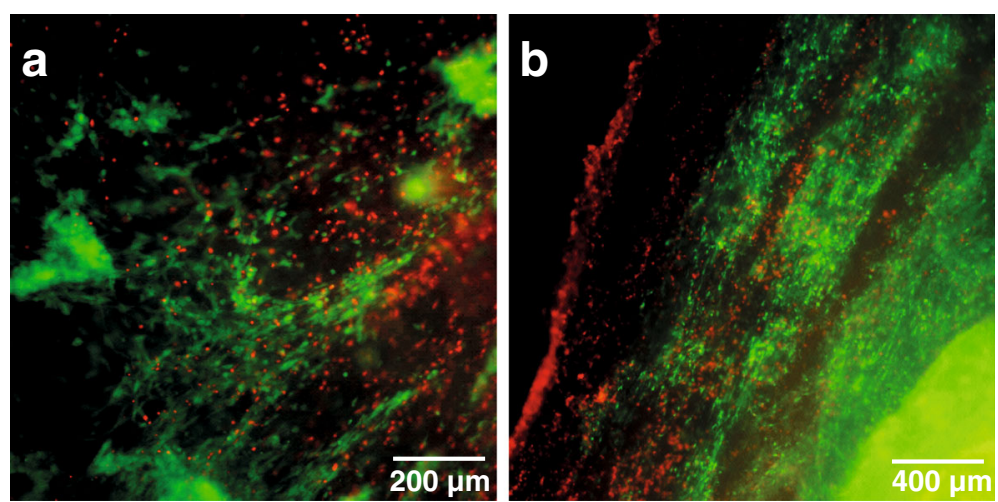

Fig. 5 Fluorescence microscopic assessment of cytocompatibility. Representative panels of LIVE/DEAD ${ }^{\circledR}$ staining of equine superficial digital flexor tendon specimens decellularized by automated (a) and manual (b) freeze thaw cycles. Decellularized scaffolds were re-seeded with equine adipose tissue derived mesenchymal stromal cells and a fluorescence microscopic evaluation was performed after 3 days of culture. Vital cells are indicated by green fluorescence (display of intracellular esterase activity), cells with defect cellular membranes show a red fluorescence signal of their nucleus

\section{Discussion}

The present study demonstrated the same high effectiveness of both automated and manual freeze-thaw cycles in decellularization of large tendon samples (equine superficial digital flexor tendon). Since the sole application of repeated freeze-thaw cycles for successful decellularization of natural tendon tissue has been reported as insufficient [10,11], all protocols applied in this study were combined with a Triton X-100-based chemical treatment. Triton $\mathrm{X}-100$ as a non-ionic detergent is commonly used in various decellularization protocols to solubilize cell membranes and dissociate DNA from proteins [15]. Since a lack of ionic charge results in a low impact on protein structures, non-ionic detergents are among the widely used chemical reagents for tissue decellularization [9]. However, there are conflicting data about the outcome of the decellularization effectiveness especially of Triton X-100 in current literature [16-23]. A direct comparison between treatment protocols particularly for decellularization of tendon tissue is difficult because of variations in the used concentrations, combinations and inconsistencies in data analysis and the times studied. Here, Triton X-100 was mainly chosen based on the results of our previous study, which had demonstrated the use of freeze-thaw cycles combined with this detergent to be effective for decellularization of full-thickness equine superficial digital flexor tendons and to maintain scaffold cytocompatibility [11].

Not only the generally high diversity in applied protocols for tendon decellularization, but also a lack of wellstudied decellularization procedures especially for equine tendon tissue complicate a methodological comparison with focus on standardization. Referring to decellularization of equine flexor tendons, there are also successful decellularization protocols applying ionic detergents (sodium dodecyl sulfate; SDS) or organic solvents (tributyl phosphate; TnBP) as chemical agents for decellularization $[23,24]$. But as far as the authors know, beside published data of Burk et al. there are only very few studies using repeated freeze-thawing for decellularization of equine flexor tendons $[11,14]$. Moreover, the latter is focused on viability and biosynthesis of tendon matrix-seeded cells, rather than on a structured evaluation of decellularization effectiveness. Finally, to the best of our knowledge, there is no methodological gold standard for decellularization of full-thickness equine superficial digital flexor tendons mentioned in the literature so far.

In the present study, we inferred no significant difference between automated and manual freeze-thaw procedures with regard to histologically visible cell nuclei count. Both procedures resulted in roughly $2 \%$ remaining nuclei only. A direct comparison of histologically assessed residual nuclei to previous studies is difficult due to different scaffold size, diverse origins of the decellularized tendon and ligament tissue samples, and differing procedural steps of decellularization. The here reported results are roughly in accordance with a $100 \%$ removal of chicken flexor digitorum profundus cells by using a protocol including Triton X-100 and peracetic acid (PAA) [18]. Further, Burk et al. showed a reduction in resident cells of $99 \%$ in decellularization of equine superficial digital flexor tendon samples when processed by freeze-thawing and Triton X-100 [11]. However, protocols including Triton X-100 did not result in a successful removal of cellular remnants and led to a disrupted tendon structure in rat tail tendons and central tendon of porcine diaphragms [21, 22]. A recently published study described decellularization of equine superficial digital flexor tendon specimens in a dimension of $10 \mathrm{~cm} \times 1.5 \mathrm{~cm} \times 0.3 \mathrm{~cm}$ by using $1 \% \mathrm{TnBP}$ in combination with $0,1,3$ or $5 \%$ PAA. The obtained results showed an increasing reduction in histologically visible cells (63 to 99\%) when treated with 0 to $5 \%$ 
PAA. However, the use of 5\% PAA led to a significant decrease of proteoglycans and alterations of the ECM, such as opened collagen fibres as well as an increased pattern of the tendon crimp [24].

Further, the present study revealed no significant differences in DNA-content by direct comparison of automated and manual protocols, with roughly $13 \%$ residual DNA in all decellularization groups. Thereby, the presented results are in accordance with current literature $[11,18,23]$. However, Pridgen et al. showed no significant reduction in DNA content by using Triton X-100 for decellularization of human flexor tendons [16]. The latter illustrates again the high diversity in published data and the resulting difficulties in comparison of decellularization protocols for tendon tissue.

To our knowledge, this study is the first attempt to partly automate the process of decellularization of tendon tissue. Whereas currently published decellularization protocols for natural tendon tissue describe manually performed techniques for freeze-thawing steps [12-14, 23, 25-27], our technique provides a viable alternative to this strategy. Due to the more standardized and optimized processing with continuous documentation, automation of repetitive freezethawing procedures appears as a vital step for the development of future decellularization protocols.

Usually, controlled rate freezers are utilized in the field of cryopreservation [28] and for experimental studies that evaluate the effects of freezing on diverse tissue and cell characteristics [29], focusing on preserving vitality in terms of storage. Therefore, these machines are designed for relatively slow cooling procedures, as required for gentle freezing of cells. This is in accordance with the here reported temperature profiles of the controlled rate freezer that showed a more precise course of temperature for slower cooling rates.

On the contrary, the present study utilized a conventional liquid nitrogen-based controlled rate freezer for physical decellularization of natural tendon tissue, for which rapid freeze-thawing is commonly used. The desired effect of freezing in terms of decellularization is a direct cell injury with minimal alteration of ECM composition. Since all steps of freeze-thawing, including cooling rate as well as the coldest set point temperature, freezing hold time, thawing rate and number of repetitions, are able to influence cell injury [30], the present study applied two different cooling and heating rates. Their direct comparison revealed no significant difference in their effectiveness for decellularization of tendon samples. A slow freezing rate, associated with extracellular ice crystal formation and a subsequent hyperosmolar shift in the extracellular environment, leads to cell dehydration as well as intracellular solute concentration, which is not always lethal for resident cells. Conversely, faster cooling rates are associated with intracellular ice crystal formation, leading almost always to cell death due to disintegrated organelles and cell membranes [30]. However, the obtained data suggest a minor importance of the applied cooling rate for decellularization effectiveness in large tendon samples. For further interpretation and especially for a comparison to freeze-thawingassociated effects in other connective tissue types, it should be noted that response to and threshold for cooling rates are cell-specific features [31, 32]. Moreover, the disrupting effects of intracellular ice crystals depend on a sufficient duration of thawing [30]. Finally, in this study, the controlled rate freezer accurately performed slow cooling and heating rates of $-20{ }^{\circ} \mathrm{C}$ per min and $+20{ }^{\circ} \mathrm{C}$ per min. As this procedure was effective for decellularization of large tendon samples, the utilized controlled rate freezer is suitable for this purpose. A possible methodological refinement is related to the requirement of liquid nitrogen. Given the problematic risk of contamination during transport or storage, the use of liquid nitrogen under GMP conditions is limited [28]. Therefore, future investigations aiming at a more extensive sample processing may benefit from alternative strategies providing cryogenic temperatures.

For further characterization of the decellularized tendon scaffolds, the present study provided a basic assessment of cytocompatibility by re-seeding the tendon surface with equine AT-MSC. In the field of tissue engineering, reseeding procedures of decellularized tendon matrices are still challenging since their dense structure makes a satisfactory cell infiltration into deeper tissue layers complicated [8]. Nevertheless, promising results of cell seeded scaffolds for tendon regeneration justify the search for more appropriate re-seeding techniques [33-36]. In the present study, tendon samples that underwent automated as well as manual freeze-thaw cycles could successfully be re-seeded. However, as reported before, only a very low number of cells penetrated deeper tissue layers, emphasizing the need for improved re-seeding techniques. The latter should aim for residual-free tendon scaffolds with a loosened matrix to allow ingrowth of scaffold-seeded cells into an almost completely preserved tissue-specific ECM. Among already published technical approaches promising effects resulted especially from using ultrasound sonication, PAA treatment and several injection techniques $[1,8,37-39]$. Further, the reduction in scaffold dimension to a thickness of $300 \mu \mathrm{m}$ led to an improved tissue penetration of cells in the present experimental set-up (unpublished data) as well as in already published studies [25]. Although the majority of cells seeded on the tendon scaffolds was vital, there were also roundly shaped, damaged cells observed by LIVE/DEAD staining in the present study. Referring to possible causes that led to damaged and detached cells, future re-seeding procedures should reduce manual handling of the seeded constructs. 
Other factors that mainly influence cell viability include chemical residues with cytotoxic effects and the initial cell seeding density. Particularly, metabolic stressors such as nutrient availability and metabolic byproducts of cellular origin gain in importance as cell density increases [40].

The horse is referred to as the fourth most frequently used source of tendon tissue for decellularization [5]. In terms of translation, the equine superficial digital flexor tendon is considered to be particularly important since its functional, structural, and pathological characteristics have already been subject of extensive research [41, 42] and are similar to those of the human Achilles tendon. Therefore, the horse is considered as the ideal animal model for human tendon pathologies, such as exerciseinduced Achilles tendon injury [43]. With regard to providing commercially available tissue grafts for xenotransplantation, equine tendon tissue offers further benefits. Those include the accessibility related to breeding and slaughter, the provided dimension of tendon tissue required in reconstructive surgery, and the limited number of zoonotic agents [5].

Future studies should aim to assess further characteristics of tendon matrices that underwent automated freeze-thaw cycles. These could include an evaluation of ECM ultrastructure and biochemical composition, of mechanical properties, of antigen removal and of adverse host reactions induced by the tendon scaffolds. To allow the production of commercially available tendon graft materials for use in human as well as in veterinary medicine, high quality manufacturing processes are required. Therefore, automation of so far manually performed key procedures in tissue decellularization, such as freeze-thawing, is essential. Moreover, future methodological research should focus on the automation of further decellularization steps. The latter especially include automated washing steps under continuous agitation performed in a closed system and applicable for a high number of samples. Finally, increased requirements with regard to validation, reproducibility, and safety, need to match with practicable approaches.

\section{Conclusion}

Automated freeze-thaw cycles carried out by a liquid nitrogen-based controlled rate freezer are effective for decellularization of large tendons when combined with a non-ionic detergent treatment (Triton X-100). These findings are an essential step towards a standardized production of decellularized tendon scaffolds for various in vitro applications and further development of graft-based reconstruction of musculoskeletal defects.

\section{Abbreviations}

AT - MSC: Adipose tissue-derived mesenchymal stromal cells;

ECM: Extracellular matrix; FBS: Fetal bovine serum; GMP: Good manufacturing practice; PAA: Peracetic acid; PBS: Phosphate buffered saline; SDS: Sodium dodecyl sulfate; TnBP: Tributyl phosphate

\section{Acknowledgements}

The authors thank Planer PLC for developing the customized sample holders and for providing the controlled rate freezer.

\section{Funding}

The work presented in this paper was made possible by funding from the German Federal Ministry of Education and Research (BMBF 1315883), the German National Research Foundation and the Saxon Ministry of Science and the Fine Arts. We acknowledge support from the German Research Foundation (DFG) and Universität Leipzig within the program of Open Access Publishing.

\section{Availability of data and materials}

The datasets supporting the conclusions of this article are available from the corresponding author on request.

\section{Authors' contributions}

SMG carried out the experiments, analysed the data and helped to draft the manuscript. AA, IE, SH helped to design the study. JB designed the study, performed the statistical analysis of the data and revised the manuscript. SPR helped to perform manual and automated decellularization, analysed the data, and wrote the manuscript. All authors read and approved the final version of the manuscript.

\section{Competing interests}

The authors declare that they have no competing interests.

\section{Consent for publication}

Not applicable.

\section{Ethics approval and consent to participate}

All of the donor animals were sacrificed for reasons unrelated to the current study. Therefore, in accordance with national guidelines and the local ethics committee (Landesdirektion Leipzig), no license was required for sample collection.

\section{Author details}

${ }^{1}$ Large Animal Clinic for Surgery, University of Leipzig, An den Tierkliniken 21, Leipzig 04103, Germany. ${ }^{2}$ Saxonian Incubator for Clinical Translation, University of Leipzig, Philipp-Rosenthal-Strasse 55, Leipzig 04103, Germany. ${ }^{3}$ Tierklinik Kaufungen, Pfingstweide 2, Kaufungen 34260, Germany.

${ }^{4}$ Department of Pathology and Laboratory Medicine, Tulane University, New Orleans, USA. ${ }^{5}$ nstitute of Veterinary Physiology, University of Leipzig, An den Tierkliniken 7, Leipzig 04103, Germany.

Received: 1 June 2016 Accepted: 3 February 2017

Published online: 14 February 2017

\section{References}

1. Cheng CW, Solorio LD, Alsberg E. Decellularized tissue and cell-derived extracellular matrices as scaffolds for orthopaedic tissue engineering. Biotechnol Adv. 2014;32:462-84.

2. Yang G, Rothrauff BB, Tuan RS. Tendon and ligament regeneration and repair: clinical relevance and developmental paradigm. Birth Defects Res C Embryo Today. 2013:99:203-22

3. Verdiyeva G, Koshy K, Glibbery N, Mann H, Seifalian AM. Tendon reconstruction with tissue engineering approach-a review. J Biomed Nanotechnol. 2015;11:1495-523.

4. Siegel L, Vandenakker-Albanese C, Siegel D. Anterior cruciate ligament injuries: anatomy, physiology, biomechanics, and management. Clin J Sport Med. 2012;22:349-55.

5. Lovati $A B$, Bottagisio $M$, Moretti $M$. Decellularized and engineered tendons as biological substitutes: a critical review. Stem Cells Int. 2016;2016:7276150.

6. Youngstrom DW, Barrett JG. Engineering tendon: scaffolds, bioreactors, and models of regeneration. Stem Cells Int. 2016;2016:3919030.

7. Crowe CS, Chattopadhyay A, McGoldrick R, Chiou G, Pham H, Chang J. Characteristics of reconstituted lyophilized tendon hydrogel: an injectable scaffold for tendon regeneration. Plast Reconstr Surg. 2016;137:843-51.

8. Schulze-Tanzil G, Al-Sadi O, Ertel W, Lohan A. Decellularized tendon extracellular matrix - a valuable approach for tendon reconstruction? Cells. 2012;1:1010-28. 
9. Gilbert TW. Strategies for tissue and organ decellularization. J Cell Biochem. 2012;113:2217-22.

10. Gilbert TW, Sellaro TL, Badylak SF. Decellularization of tissues and organs. Biomaterials. 2006;27:3675-83.

11. Burk J, Erbe I, Berner D, Kacza J, Kasper C, Pfeiffer B, et al. Freeze-thaw cycles enhance decellularization of large tendons. Tissue Eng Part C Methods. 2014;20:276-84

12. Azuma C, Tohyama H, Nakamura H, Kanaya F, Yasuda K. Antibody neutralization of TGF- $\beta$ enhances the deterioration of collagen fascicles in a tissue-cultured tendon matrix with ex vivo fibroblast infiltration. J Biomech. 2007:40:2184-90.

13. Omae H, Zhao C, Sun YL, An K-N, Amadio PC. Multilayer tendon slices seeded with bone marrow stromal cells: a novel composite for tendon engineering. J Orthop Res. 2009;27:937-42.

14. Stewart AA, Barrett JG, Byron CR, Yates AC, Durgam SS, Evans RB, Stewart MC. Comparison of equine tendon-, muscle-, and bone marrow-derived cells cultured on tendon matrix. Am J Vet Res. 2009;70:750-7.

15. Crapo PM, Gilbert TW, Badylak SF. An overview of tissue and whole organ decellularization processes. Biomaterials. 2011;32:3233-43.

16. Pridgen BC, Woon CYL, Kim M, Thorfinn J, Lindsey D, Pham H, Chang J. Flexor tendon tissue engineering: acellularization of human flexor tendons with preservation of biomechanical properties and biocompatibility. Tissue Eng Part C Methods. 2011:17:819-28.

17. Vavken P, Joshi S, Murray MM. TRITON-X is most effective among three decellularization agents for ACL tissue engineering. J Orthop Res. 2009;27:1612-8.

18. Whitlock PW, Smith TL, Poehling GG, Shilt JS, van Dyke M. A naturally derived, cytocompatible, and architecturally optimized scaffold for tendon and ligament regeneration. Biomaterials. 2007;28:4321-9.

19. Harrison RD, Gratzer PF. Effect of extraction protocols and epidermal growth factor on the cellular repopulation of decellularized anterior cruciate ligament allografts. J Biomed Mater Res A. 2005;75:841-54.

20. Woods $T$, Gratzer PF. Effectiveness of three extraction techniques in the development of a decellularized bone-anterior cruciate ligament-bone graft. Biomaterials. 2005;26:7339-49.

21. Cartmell JS, Dunn MG. Effect of chemical treatments on tendon cellularity and mechanical properties. J Biomed Mater Res. 2000;49:134-40.

22. Deeken CR, White AK, Bachman SL, Ramshaw BJ, Cleveland DS, Loy TS, Grant SA. Method of preparing a decellularized porcine tendon using tributyl phosphate. J Biomed Mater Res B Appl Biomater. 2011;96:199-206.

23. Youngstrom DW, Barrett JG, Jose RR, Kaplan DL. Functional characterization of detergent-decellularized equine tendon extracellular matrix for tissue engineering applications. PLoS One. 2013;8:e64151.

24. Bottagisio M, Pellegata AF, Boschetti F, Ferroni M, Moretti M, Lovati AB. A new strategy for the decellularisation of large equine tendons as biocompatible tendon substitutes. Eur Cell Mater. 2016;32:58-73.

25. Ning $L$, Zhang $Y$, Chen $X H$, Luo JC, Li XQ, Yang ZM, Qin TW. Preparation and characterization of decellularized tendon slices for tendon tissue engineering. J Biomed Mater Res A. 2012;100:1448-56.

26. Ning $L$ J, Zhang $Y J$, Zhang $Y$, Qing $Q$, Jiang $Y L$, Yang $J$, et al. The utilization of decellularized tendon slices to provide an inductive microenvironment for the proliferation and tenogenic differentiation of stem cells. Biomaterials. 2015;52:539-50.

27. Durgam SS, Stewart AA, Pondenis HC, Gutierrez-Nibeyro SM, Evans RB, Stewart MC. Comparison of equine tendon- and bone marrow-derived cells cultured on tendon matrix with or without insulin-like growth factor-I supplementation. Am J Vet Res. 2012;73:153-61.

28. Massie I, Selden C, Hodgson H, Fuller B, Gibbons S, Morris GJ. GMP cryopreservation of large volumes of cells for regenerative medicine: active control of the freezing process. Tissue Eng Part C Methods. 2014:20:693-702.

29. Devireddy RV, Neidert, Bischof JC, Tranquillo RT. Cryopreservation of collagen-based tissue equivalents. I. Effect of freezing in the absence of cryoprotective agents. Tissue Eng. 2003;9:1089-100.

30. Gage AA, Baust J. Mechanisms of tissue injury in cryosurgery. Cryobiology. 1998:37:171-86

31. Yiu WK, Basco MT, Aruny JE, Cheng SW, Sumpio BE. Cryosurgery: a review. Int J Angiol. 2007;16:1-6.

32. Suto K, Urabe K, Naruse K, Uchida K, Matsuura T, Mikuni-Takagaki Y, et al. Repeated freeze-thaw cycles reduce the survival rate of osteocytes in bone-tendon constructs without affecting the mechanical properties of tendons. Cell Tissue Bank. 2012:13:71-80.
33. Gungormus C, Kolankaya D, Aydin E. Histopathological and biomechanica evaluation of tenocyte seeded allografts on rat Achilles tendon regeneration. Biomaterials. 2015;51:108-18.

34. Whitlock PW, Seyler TM, Parks GD, Ornelles DA, Smith TL, van Dyke ME, Poehling GG. A novel process for optimizing musculoskeletal allograft tissue to improve safety, ultrastructural properties, and cell infiltration. J Bone Joint Surg Am. 2012;94:1458-67.

35. Liu W, Chen B, Deng D, Xu F, Cui L, Cao Y. Repair of tendon defect with dermal fibroblast engineered tendon in a porcine model. Tissue Eng. 2006;12:775-88.

36. Yin Z, Chen X, Zhu T, Hu JJ, Song HX, Shen WL, et al. The effect of decellularized matrices on human tendon stem/progenitor cell differentiation and tendon repair. Acta Biomater. 2013;9:9317-29.

37. Ingram JH, Korossis S, Howling G, Fisher J, Ingham E. The use of ultrasonication to aid recellularization of acellular natural tissue scaffolds for use in anterior cruciate ligament reconstruction. Tissue Eng. 2007;13:1561-72.

38. Martinello T, Bronzini I, Volpin A, Vindigni V, Maccatrozzo L, Caporale G, et al. Successful recellularization of human tendon scaffolds using adiposederived mesenchymal stem cells and collagen gel. J Tissue Eng Regen Med. 2012;8:612-9.

39. Woon CYL, Pridgen BC, Kraus A, Bari S, Pham H, Chang J. Optimization of human tendon tissue engineering: peracetic acid oxidation for enhanced reseeding of acellularized intrasynovial tendon. Plast Reconstr Surg. 2011; 127:1107-17.

40. Issa RI, Engebretson B, Rustom L, McFetridge PS, Sikavitsas VI. The effect of cell seeding density on the cellular and mechanical properties of a mechanostimulated tissue-engineered tendon. Tissue Eng Part A. 2011;17: 1479-87.

41. Thorpe CT, Clegg PD, Birch HL. A review of tendon injury: Why is the equine superficial digital flexor tendon most at risk? Equine Vet J. 2010;42: $174-80$.

42. Dowling BA, Dart AJ. Mechanical and functional properties of the equine superficial digital flexor tendon. Vet J. 2005;170:184-92.

43. Patterson-Kane JC, Rich T. Achilles tendon injuries in elite athletes: lessons in pathophysiology from their equine counterparts. ILAR J. 2014:55:86-99.

\section{Submit your next manuscript to BioMed Central and we will help you at every step:}

- We accept pre-submission inquiries

- Our selector tool helps you to find the most relevant journal

- We provide round the clock customer support

- Convenient online submission

- Thorough peer review

- Inclusion in PubMed and all major indexing services

- Maximum visibility for your research

Submit your manuscript at www.biomedcentral.com/submit
) Biomed Central 